\title{
Some Property of Finite Sum of Weighted Composition and Weighted Frobenius-Perron Operators
}

\author{
Abolghasem Alishahi \\ Department of Mathematics, Payame Noor University, Tehran, Iran \\ Email address: \\ A_alishahy@pnu.ac.ir \\ To cite this article: \\ Abolghasem Alishahi. Some Property of Finite Sum of Weighted Composition and Weighted Frobenius-Perron Operators. International \\ Journal of Theoretical and Applied Mathematics. Vol. 5, No. 4, 2019, pp. 63-67. doi: 10.11648/j.ijtam.20190504.12
}

Received: May 26, 2019; Accepted: July 1, 2019; Published: October 25, 2019

\begin{abstract}
The study of finite sum of weighted composition operators on $L^{p}$ - spaces has received considerable attention in 2012. Characterizations, basic properties of this operators have been obtained. Weighted composition operators are a general class of operators and they appear naturally in the study of surjective isometries on most of the function spaces, semi grup theory, dynamic systems etc. This type of operators are a generalization of multiplication operators and composition operators. In this paper the relations among completely continuous and $\mathrm{M}$ - weakly compact of finite sum of weighted composition operators between $L^{p}(\mu)$ - spaces described, we also obtain some necessary and sufficient conditions for Fredholmness of the finite sum of weighted composition operators.
\end{abstract}

Keywords: Completely Continuous, M - weakly Compact, Fredholm Operators, Absolutely Continuous, Conditional Expectation Operator

\section{Introduction}

Weighted composition operators are a general class of operators. There are many great papers on the investigation of weighted composition operators acting on the spaces of measurable functions. For instance, [10-15]. The finite sum of weighted composition operators were studied on $L^{p}$-spaces [9, 15]. The basic property of weighted composition operators of measurable function spaces are studied by Lambert $[1,2]$, Singh and Manhas [5], Takagi [6] and sum other mathematicians. Let $(\mathrm{X}, \Sigma, \mu)$ be a complete $\sigma$-finite measure space and suppose $u_{i}: \mathrm{X} \rightarrow \mathbb{C}$ is a $\Sigma$ - measurable function and $\varphi_{i}: \mathrm{X} \rightarrow \mathbb{C}$ be a non singular measurable transformation, i. e the measure $\mu o \varphi_{i}^{-1}$ is absolutely continuous with respect to the $\mu$, where $\mu \circ \varphi_{i}^{-1}(A)=\mu\left(\varphi_{i}^{-1}(A)\right)$ for all $A \in \Sigma$ For any $f \in L^{p}(\mu)$, by the Radon-Nikodym theorem, there exists a unique $\varphi_{i}^{-1}(\Sigma)$-measurable function $E^{i}$ such that $\int_{A} E^{i} f d \mu=\int_{A} f d \mu$ for all $A \in \Sigma$. Hence we obtain an operator $E^{i}$ which is called conditional expectation operator. If $A$ is any $\Sigma$-measurable set for which $\int_{\varphi_{i}{ }^{-1}(A)} u_{i} f d \mu$ exists, the linear operator $\mathcal{P}_{\varphi_{i}}^{u_{i}}: L^{1}(\mu) \rightarrow L^{1}(\mu)$ define by $\int_{A} \mathcal{P}_{\varphi_{i}}^{u_{i}} f d \mu=\int_{\varphi_{i}{ }^{-1}(A)} u_{i} f d \mu$ is called Weighted Frobenius-Perron associated with the pair $\left(u_{i}, \varphi_{i}\right)$.
In this paper we consider finite sum of weighted composition operators and weighted Frobeninus-Perron operators defined on $L^{p}(\Sigma)$ and $L^{1}(\Sigma)$ respectively of the form

$$
W=\sum_{i=1}^{n} u_{i} C_{\varphi_{i}}, \mathcal{P}=\sum_{i=1}^{n} \mathcal{P}_{\varphi_{i}}^{u_{i}}
$$

Also we give some sufficient and necessary conditions for completely continuous, $M$-weakly compact and Fredholmness of finite sum of weighted composition operator $W$ on $L^{p}(\Sigma)$ and finite sum of weighted Frobeniuse-Perron operator $\mathcal{P}$ on $L^{1}(\Sigma)$.

\section{Completely Continuous and $M$-weakly Compact}

We recall the definitions of completely Continuous and $M$ - weakly compact:

Definition 2.1

Let $B_{1}$ and $B_{2}$ be two Banach spaces over $\mathbb{C}$. A bounded linear operator $T: B_{1} \rightarrow B_{2}$ is said to be completely continuous if it maps weakly convergent sequences in $B_{1}$ into strongly convergent sequences in $B_{2}$. In the sequal, we adopt the following decomposition of $(\mathrm{X}, \Sigma, \mu)$ : 


$$
X=\left(\bigcup_{i=1}^{n} A_{i}\right) \cup B
$$

where $\left(A_{i}\right)_{i \in \mathbb{N}}$ is acountable collection of pairwise disjoint atoms and $B$, being disjoint from each $A_{i}$, is non - atomic.

Definition 2.2

A bounded linear operator $T: L^{p}(\mu) \rightarrow L^{q}(\mu)$, where $1 \leq p, q \leq \infty \quad, \quad$ is $M \quad$ - weakly compact if $\lim _{n \rightarrow \infty}\left\|T f_{n}\right\|_{L^{q}(\mu)}=0$, for every bounded sequence $\left(f_{n}\right)_{i \in \mathbb{N}}$ in $L^{p}(\mu)$ such that $\mu\left(\operatorname{Coz} f_{m} \cap \operatorname{Coz} f_{n}\right)=0$ whenever $m \leq n$. This type of operators was introduced by P. Meyer - Nieberg [1] in the study of Riesz spaces.

In this theorem we give some necessary and sufficient conditions for $W$ to be completely continuous as an operator from $L^{1}(\mu)$ into $L^{1}(\mu)$.

Theorem 2.1

Let $W$ be a finite sum of weighted composition operators from $L^{1}(\mu)$ into $L^{1}(\mu)$ and $J \in L^{\infty}(\mu)$ where $J=$ $\sum_{i=1}^{n} h_{i} E^{i}\left(\left|u_{i}\right|^{p}\right) o \varphi_{i}^{-1}$. If $J(B)=0, \mu-$ a.e then $W$ is completely continuous.

Proof

We suppose that $J(B)=0, \mu-$ a. e. Let $\left(f_{n}\right)_{i \in \mathbb{N}}$ be an arbitrary sequence in $L^{1}(\mu)$ such that $f_{n} \rightarrow 0$ weakly. Putting $A=\bigcup_{i=1}^{n} A_{i}$. We have

$$
\left\|W f_{n}\right\|_{L^{1}(\mu)}=\int_{X}\left|W f_{n}\right| d \mu \leq \sum_{i=1}^{n} \int_{X} J_{i}\left|f_{n}\right| d \mu=\int_{A} J\left|f_{n}\right| d \mu+\int_{B} J\left|f_{n}\right| d \mu=\int_{X} J\left|f_{n}\right| \chi_{A} d \mu \leq|| J||_{L^{\infty}(\mu)}|| f_{n} \chi_{A}||_{L^{1}(\mu)}
$$

From the weak convergence of the sequence $\left(f_{n}\right)_{i \in \mathbb{N}}$, we see that $f_{n}\left(A_{i}\right) \rightarrow 0$ for each fixed $i \in \mathbb{N}$. Thus $f_{n} \chi_{A} \rightarrow$ $0, \mu-$ a.e on $\mathrm{X}$. It follows that the sequence $\left(f_{n} \chi_{A}\right)_{n \in \mathbb{N}}$ converges in $\mu$-measure. This, together with the fact that $f_{n} \chi_{A} \rightarrow 0$ weakly in $L^{1}(\mu)$. Yields $\left\|f_{n} \chi_{A}\right\|_{L^{1}(\mu)} \rightarrow$ 0 [4]. Hence $W$ is completely continuous.

In the next theorem we give some necessary and sufficient conditions for $W$ to be compact as an operator from $L^{\infty}(\mu)$ into $L^{\infty}(\mu)$.

Theorem 2.2

Let $u=\sum_{i=1}^{n}\left|u_{i}\right|, N_{\varepsilon}(u)=\{x \in X:|u(x)| \geq \varepsilon\}$ and $W$ be a bounded operator from $L^{\infty}(\mu)$ into $L^{\infty}(\mu)$. Then the followings hold.

(a) If for each $\varepsilon, N_{\varepsilon}(u)$ consists of finitely many atoms then $W$ is a compact operator from $L^{\infty}(\mu)$ into $L^{\infty}(\mu)$. (b) If $W$ is a compact operator from $L^{\infty}(\mu)$ into $L^{\infty}(\mu)$ then $W$ is $M$-weakly compact.

(c) Let $(\mathrm{X}, \Sigma, \mu)$ be a finite measure space and the sequence $\left\{\mu\left(A_{i}\right)\right\}_{i \in \mathbb{N}}$ has no subsequence that converges to zero. If $u_{i}$ 's are non-negative, $1<p<\infty$ and for each $\varepsilon>0, \sum_{i=1}^{n} u_{i}{ }^{p} \geq \varepsilon$ then $W$ is a compact operator from $L^{\infty}(\mu)$ into $L^{\infty}(\mu)$ if and only if for each $\varepsilon>0, N_{\varepsilon}(u)$ consists of finitely many atoms.

Proof

(a) Let $\varepsilon>0$ and $A=N_{\varepsilon}(u)=\bigcup_{j=1}^{k} A_{j}^{\varepsilon}$, where $A_{j}^{\varepsilon}$ 's are disjoint atoms. Suppose that $W^{\prime}=W M_{\chi_{A}}$ where $M_{\chi_{A}} f=\sum_{j=1}^{k} f\left(A_{j}^{\varepsilon}\right) \chi_{A_{j}^{\varepsilon}}$. It follows that $W^{\prime}$ is finite rank operator on $L^{\infty}(\mu)$. Hence for every $f \in$ $L^{\infty}(\mu)$, we have

$$
\left|W^{\prime} f-W f\right|=\left|\sum_{i=1}^{n} u_{i} f o \varphi_{i} \chi_{X \backslash A} O \varphi_{i}\right| \leq \sum_{i=1}^{n}\left|u_{i}\right|\left|f o \varphi_{i}\right| \chi_{X \backslash A} O \varphi_{i} \leq \varepsilon|| f||_{L^{\infty}(\mu)}
$$

Thus we get that $\left\|W^{\prime} f-W f\right\| \leq \varepsilon$ and so $W$ is compact.

(b) Choose any bounded sequence $\left(f_{n}\right)_{i \in \mathbb{N}}$ in $L^{p}(\mu)$ such that $\mu\left(\operatorname{Coz} f_{m} \cap \operatorname{Coz} f_{n}\right)=0$ whenever $m \leq n$. Let $S=\bigcup_{m<n \in \mathbb{N}}\left(\operatorname{Coz} f_{m} \cap \operatorname{Coz} f_{n}\right)$. Then, $\mu(S)=0$ and at most one $f_{n}$ satisfies the condition that $f_{n}(x) \neq 0$ for every $x \in X \backslash S$. Therefor, $f_{n}(x) \rightarrow 0$, $\mu-$ a. e on $X$. To prove the desired result, we assume the contrary that there exists a constant $\varepsilon_{0}>0$ with $\left\|W f_{n}\right\|_{L^{\infty}(\mu)} \geq \varepsilon_{0}$ for all $n$. Sinc $W$ is compact, there exists a subsequence $\left(f_{n_{k}}\right)_{k \in \mathbb{N}}$ and a function $g$ in $L^{\infty}(\mu)$ such that $\left\|W f_{n_{k}}-g\right\| \rightarrow 0$ as $k \rightarrow 0$. Then we have that $\lim _{k \rightarrow \infty} W f_{n_{k}}(x)=g(x), \mu-$ a.e on $X$. So $\left\|W f_{n}\right\|_{L^{\infty}(\mu)} \rightarrow 0$. This is contradiction.

(c) By part (a) we only show that $W$ is compact operator then for each $\varepsilon>0, N_{\varepsilon}(u)$ consists of finitely many atoms. Suppose on the contrary. Then there exists $\varepsilon>0$ such that $N_{\varepsilon}(u)$ contains infinitely many atoms. Let $\left\{A_{j}\right\}_{i \in \mathbb{N}}$ be disjoint atoms in $N_{\varepsilon}(u)$. Put $g_{j}=$ $\frac{\chi_{A_{j}}}{\sqrt[p]{\mu\left(A_{j}\right)}}$. Let $A \in \Sigma$ with $0<\mu(A)<\infty$. Since sequence $\left\{\mu\left(A_{j}\right)\right\}_{i \in \mathbb{N}}$ has no subsequence that converges to zero, then $\left\{j ; A_{j} \subseteq A\right.$ is finite and so $\mu\left(A_{j} \cap A\right)=0$ for sufficiently large $j$ and
$\left|\int_{X} g_{j} \chi_{A} d \mu\right|=\frac{\mu\left(A_{j} \cap A\right)}{\sqrt[p]{\mu\left(A_{j}\right)}} \rightarrow 0$ as $j \rightarrow \infty$. It follows that

$g_{j} \rightarrow \infty$ weakly. Since $W$ is compact it follows that $\left\|W g_{j}\right\|_{\infty} \rightarrow 0$ On the other hand we have

$$
\begin{gathered}
|| W g_{j}||_{\infty}^{p} \mu(X) \geq|| W g_{j}||_{p}^{p}=\int_{X}\left|\sum_{i=1}^{n} u_{i} \frac{\chi_{A_{j} o \varphi_{i}}}{\sqrt[p]{\mu\left(A_{j}\right)}}\right|^{p} d \mu \\
\geq \sum_{i=1}^{n} \int_{X} u_{i}^{p} \frac{\chi_{A_{j}} o \varphi_{i}}{\mu\left(A_{j}\right)} d \mu \\
=\sum_{i=1}^{p} u_{i}^{p} \\
\geq \varepsilon
\end{gathered}
$$

Thus we get that $\left\|W g_{j}\right\|_{\infty} \geq \sqrt[p]{\frac{\varepsilon}{\mu(X)}}$. But this is a contradiction.

\section{Fredholmness of the Finite Sum of Wieghted Composition Operators}

In this section we assume $1 \leq p<\infty$. Ths special case of 
Fredholm weighted composition operators $u C_{\varphi}$ has been studied in the study [3]. We will prove the general case for the finite sum of weighted composition operator $W$.

\section{Lemma 3.1}

Suppose $(X, \Sigma, \mu)$ is non - atomic, $u_{i}\left(\varphi_{j}\right)=0, i \neq j$ and let $W$ be a bounded operator from $L^{p}(\mu)$ into $L^{p}(\mu)$.

(a) The nullity of $W$ (i. e. $\operatorname{dim} \operatorname{ker} W$ ) is either zero or infinite.

(b) The codimention of $\overline{\operatorname{ran}(W)}$ in $L^{p}(\mu)$ (i.e. $\left.\operatorname{dim} \frac{L^{p}(\mu) .}{\overline{r a n(W)}}\right)$ is either zero or infinite.

Proof

We first prove (a). If $W$ is injective, then $\operatorname{dim} \operatorname{ker} W=0$. Otherwise, there a non - zero function $f \in L^{p}(\mu)$ such that $W f=0$. As $(X, \Sigma, \mu)$ is non - atomic and the set $E:=\{x \in$ $X:|f(x)|>0\}$ is of positive $\mu$ - measure, we may choose a sequence $\left\{E_{n}\right\}_{n=1}^{\infty}$ of pairwise disjoint, $\Sigma$ - measurable sets in $E$ with $0<\mu\left(E_{n}\right)<\infty$. Let $f_{n}:=f \chi_{n}$ for $n \in \mathbb{N}$. They are non - zero and linearly independent. Moreover,

$$
\begin{aligned}
|| W f_{n}||_{L^{p}(\mu)}^{p} & =\int_{X}\left|\sum_{i=1}^{n} u_{i} f_{n} o \varphi_{i}\right|^{p} d \mu=\int_{X}\left|\sum_{i=1}^{n} u_{i} f o \varphi_{i} \chi_{E_{n}} o \varphi_{i_{i}}\right|^{p} d \mu \\
& =\int_{X}\left|\left(\sum_{i=1}^{n} u_{i} f o \varphi_{i}\right)\left(\sum_{i=1}^{n} \chi_{E_{n}} o \varphi_{i}\right)-\sum_{i \neq j} u_{i} f o \varphi_{i} \chi_{E_{n}} o \varphi_{j}\right|^{p} d \mu
\end{aligned}
$$

So

$$
\begin{aligned}
|| W f_{n} \|_{L^{p}(\mu)}^{p} & \leq 2^{p-1} \int_{X}\left|\left(\sum_{i=1}^{n} u f o \varphi_{i}\right)\left(\sum_{i=1}^{n} \chi_{E_{n}} o \varphi_{i}\right)\right|^{p} d \mu+2^{p-1} \int_{X}\left|\sum_{i \neq j} u_{i} f o \varphi_{i} \chi_{E_{n}} o \varphi_{j}\right|^{p} d \mu \\
& =2^{p-1} \int_{X}\left|\sum_{i=1}^{n} u f o \varphi_{i_{i}}\right|^{p}\left(\sum_{i=1}^{n} \chi_{E_{n}} o \varphi_{i}\right)^{p} d \mu+2^{p-1} \int_{X}\left|\sum_{i \neq j} u_{i} f o \varphi_{i} \chi_{E_{n}} o \varphi_{j}\right|^{p} d \mu
\end{aligned}
$$

Then

$$
\begin{aligned}
& || W f_{n}||_{L^{p}(\mu)}^{p} \leq 2^{p-1} \int_{X}|W f|^{p} \sum_{k_{1}+\cdots+k_{n}=p, k_{i} \geq 0}\left(\begin{array}{c}
p \\
k_{1}, \ldots, k_{n}
\end{array}\right) \chi_{E_{1}}{ }^{k_{1}} o \varphi_{i} \ldots \chi_{E_{n}}{ }^{k_{n}} o \varphi_{n} d \mu+2^{p-1} \int_{X}\left|\sum_{i \neq j} u_{i} f o \varphi_{i} \chi_{E_{n}} o \varphi_{j}\right|^{p} d \mu \\
& \leq 2^{p-1} \sum_{k_{1}+\cdots+k_{n}=p, k_{i} \geq 0}\left(\begin{array}{c}
p \\
k_{1}, \ldots, k_{n}
\end{array}\right) \int_{X}|W f|^{p} \chi_{E_{1}} o \varphi_{i} \ldots \chi_{E_{n}} o \varphi_{n} d \mu+2^{p-1}(n(n-1))^{p-1} \sum_{i \neq j} \int_{X}\left|u_{i} f o \varphi_{i}\right|^{p} \chi_{E_{n}} o \varphi_{j} d \mu
\end{aligned}
$$

And so

$$
\left.|| W f_{n}\right|_{L^{p}(\mu)} ^{p} \leq 2^{p-1} \sum_{k_{1}+\cdots+k_{n}=p, k_{i} \geq 0}\left(\begin{array}{c}
p \\
k_{1}, \ldots, k_{n}
\end{array}\right) \int_{\cap_{i=1}^{n} \varphi_{i}^{-1}\left(E_{n}\right)}\left|W f_{i}\right|^{p} d \mu+2^{p-1}(n(n-1))^{p-1} \sum_{i \neq j} \int_{\varphi_{j}^{-1}\left(E_{n}\right)}\left|u_{i} f o \varphi_{i}\right|^{p} \chi_{E_{n}} o \varphi_{j} d \mu
$$

So

$$
\begin{gathered}
|| W f_{n} \|_{L^{p}(\mu)}^{p} \leq 2^{p-1} \sum_{k_{1}+\cdots+k_{n}=p, k_{i} \geq 0}\left(\begin{array}{c}
p \\
k_{1}, \ldots, k_{n}
\end{array}\right) \int_{\cap_{i=1}^{n} \varphi_{i}^{-1}\left(E_{n}\right)}\left|W f_{i}\right|^{p}+2^{p-1}(n(n-1))^{p-1} \sum_{i \neq j} \int_{\varphi_{j}^{-1}\left(E_{n}\right)}\left|u_{i} f o \varphi_{i}\right|^{p} \chi_{E_{n}} o \varphi_{j} d \mu \\
\left|u_{i}\left(\varphi_{j}^{-1}\left(E_{n}\right)\right) \| f o \varphi_{i}\left(\varphi_{j}^{-1}\left(E_{n}\right)\right)\right|^{p} \mu\left(\varphi_{j}^{-1}\left(E_{n}\right)\right)
\end{gathered}
$$

Then

$$
\left\|W f_{n}\right\|_{L^{p}(\mu)}^{p} \leq 2^{p-1} \sum_{k_{1}+\cdots+k_{n}=p, k_{i} \geq 0}\left(\begin{array}{c}
p \\
k_{1}, \ldots, k_{n}
\end{array}\right) \int_{\cap_{i=1}^{n} \varphi_{i}^{-1}\left(E_{n}\right)}\left\|W f_{i}\right\|^{p} d \mu+0=0 .
$$

So that $f_{n} \in \operatorname{ker} W$ for all $n$. In this case, we have $\operatorname{dim} \operatorname{ker} W=\infty$.

For (b) we suppose that $\operatorname{dim} \frac{L^{p}(\mu)}{\overline{\operatorname{ran}(W)}} \neq 0$. As $\operatorname{dim} \frac{L^{p}(\mu)}{\operatorname{ran}(W)}=$ $\operatorname{dim} W^{*}$, there is a non-zero function $g \in L^{p}(\mu)$ (where $\left.\frac{1}{p}+\frac{1}{q}=1\right)$ such that $W^{*} f=0$ or $\int(W f) \bar{g} d \mu=0$ for all $f \in L^{p}(\mu)$. Set $E:=\{x \in X:|f(x)|>0\}$. Since $(X, \Sigma, \mu)$ is non - atomic, there exists a sequence $\left\{E_{n}\right\}_{n=1}^{\infty}$ of pairwise disjoint, $\Sigma$-measurable sets in $E$ with $0<\mu\left(E_{n}\right)<\infty$. Let $g_{n}:=g \chi_{n}$ for $n \in \mathbb{N}$. Moreover, we have

$g_{n}(W f)=\int_{X}(W f) g_{n} d \mu=\int_{E_{n}}(W f) g d \mu \leq$ $\int_{X}(W f) \bar{g} d \mu=0$ for evry $f \in L^{p}(\mu)$, i.e. $g_{n} \in \operatorname{ker} W^{*}$.
Hence $\operatorname{dim} \operatorname{ker} \frac{L^{p}(\mu)}{\operatorname{ran}(W)}=\infty$.

\section{Lemma 3.2}

Suppose $(X, \Sigma, \mu)$ is a non - atomic rich measure space, $u_{i}\left(\varphi_{j}\right)=0, i \neq j$ and let $\mathcal{P}$ be a bounded operator from $L^{1}(\mu)$ into $L^{1}(\mu)$. Then codimention of $\overline{\operatorname{ran}(\mathcal{P})}$ in $L^{1}(\mu)$ (i.e. $\left.\operatorname{dim} \frac{L^{1}(\mu)}{\overline{\operatorname{ran}(\mathcal{P})}}\right)$ is either zero or infinite.

Proof

we suppose that $\operatorname{dim} \frac{L^{1}(\mu)}{\overline{\operatorname{ran}(\mathcal{P})}} \neq 0$. As $\operatorname{dim} \frac{L^{1}(\mu)}{\overline{\operatorname{ran}(\mathcal{P})}}=$ $\operatorname{dim} \mathcal{P}^{*}=\operatorname{dim} W$, there is a non-zero function $g \in L^{\infty}(\mu)$ such that $W f=0$ or $\int(W f) \bar{g} d \mu=0$ for all $f \in L^{\infty}(\mu)$. Set $E:=\{x \in X:|f(x)|>0\}$. Since $(X, \Sigma, \mu)$ is non - 
atomic, there exists a sequence $\left\{E_{n}\right\}_{n=1}^{\infty}$ of pairwise disjoint, $\Sigma$ - measurable sets in $E$ with $0<\mu\left(E_{n}\right)<\infty$. Let $g_{n}:=g \chi_{n}$ for $n \in \mathbb{N}$. Moreover, we have

$\left\|W g_{n}\right\|_{L_{(X)}^{\infty}} \leq$

||$W f||_{L^{\infty}\left(\cup_{i=1}^{n} \varphi_{i}^{-1}\left(E_{n}\right)\right)}+\left.\sum_{i \neq j}|| u_{i} f o \varphi_{i}\right|_{L^{\infty}\left(\varphi_{j}^{-1}\left(E_{n}\right)\right)} \leq$

$\| W f||_{L^{\infty}(X)}+0=0$ for evry $f \in L^{p}(\mu)$, i.e. $g_{n} \in \operatorname{ker} W$. Hence $\operatorname{dim} \operatorname{ker} \frac{L^{1}(\mu)}{\overline{\operatorname{ran}(\mathcal{P})}}=\infty$.

Theorem 3.1

Suppose $(X, \Sigma, \mu)$ is non - atomic and $W$ be a Fredholm operator from $L^{p}(\mu)$ into $L^{p}(\mu)$. Then there exists a constant $\delta>0$ on $E$ such that $J \geq \delta$ for every set $E \in \Sigma$ with $\mu(E)<\infty$, where $J=\sum_{i=1}^{n} h_{i} E^{i}\left(\left|u_{i}\right|^{p}\right) o \varphi_{i}{ }^{-1}$. Morever if $\mu(X)<\infty$ then $J \geq \delta$ on $X$.

Proof

Assume $W$ is Fredholm. It is injective by Lemma 2.2. Since the range of $W$ is closed, there is a number $c>0$ such that

$$
\|W f\|_{p} \geq c\|f\|_{p} \text { for all } f \in L^{p}(\mu) .
$$

In particular choosing $f=\chi_{E}$ and $\delta=\frac{c^{p}}{n^{p-1}}$, where $E \in \Sigma$ and $\mu(E)<\infty$, we obtain

$$
\begin{aligned}
& { }^{p} \mu(E) \leq|| W \chi_{E}||_{p}^{p} \\
& \leq \int_{X}\left|\sum_{i=1}^{n} u_{i} \chi_{E} o \varphi_{i}\right|^{p} d \mu \\
& \leq n^{p-1} \sum_{i=1}^{n}\left|u_{i}\right|^{p} \chi_{E} o \varphi_{i} d \mu \\
& =n^{p-1} \int_{X} J \chi_{E} d \mu .
\end{aligned}
$$

Theorem 3.2

Suppose $(X, \Sigma, \mu)$ is non - atomic and $\mathcal{P}$ be a Fredholm operator from $L^{1}(\mu)$ into $L^{1}(\mu)$. Then there exists a constant $\delta>0$ on $F$ such that $\sum_{i=1}^{n}\left|u_{i}\right| \geq \delta$ for every set $F \in \Sigma$ with $\mu(F)<\infty$.

Proof

Assume $\mathcal{P}$ is Fredholm. It is injective by Lemma 3.2. Since the range of $\mathcal{P}$ is closed, there is a number $c>0$ such that

$$
\|W f\|_{L^{1}(\mu)} \geq c\|f\|_{L^{1}(\mu)} \text { for all } f \in L^{1}(\mu) .
$$

In particular choosing $f=\chi_{F}$ and $\delta=c$, where $F \in \Sigma$ and $\mu(F)<\infty$, we obtain

$$
\begin{aligned}
& c \mu(F) \leq\left\|\mathcal{P} \chi_{F}\right\|_{L^{1}(\mu)} \\
& \quad\|W f\|_{2}=\left(W^{*} W f, f\right)=\int_{X} J|f|^{2} d \mu=\int_{\text {Coz J }} J|f|^{2} d \mu+\int_{\left.\right|_{X \backslash C o Z J} J|f|^{2} d \mu \geq \delta\|f\|_{2}}
\end{aligned}
$$

$$
\begin{gathered}
=\int_{X}\left|\sum_{i=1}^{n} E^{i}\left(u_{i} \chi_{F}\right)\right| d \mu \\
\leq \int_{X} \sum_{i=1}^{n} E^{i}\left(\left|u_{i}\right| \chi_{F}\right) d \mu \\
=\int_{X} \sum_{i=1}^{n}\left|u_{i}\right| \chi_{F} d \mu \\
=\int_{F} \sum_{i=1}^{n}\left|u_{i}\right| d \mu .
\end{gathered}
$$

Theorem 3.3

Suppose $(X, \Sigma, \mu)$ is non - atomic and $W$ be a bounded operator from $L^{2}(\mu)$ into $L^{2}(\mu)$. The following statements holds:

(a) If $W$ be a Fredholm operator then there exists a constant $\delta>0$ on $E$ such that $J \geq \delta$ for every set $E \in \Sigma \quad$ with $\quad \mu(E)<\infty \quad$ where $J=\sum_{i=1}^{n} h_{i} E^{i}\left(\left|u_{i}\right|^{2}\right) o \varphi_{i}^{-1}$.

(b) If there exists a constant $\delta>0$ on $E$ such that $J \geq \delta$ for every set $E \in \Sigma$ with $\mu(E)<\infty$ then $W$ be a semi-Fredholm operator.

Proof

(a) Assume $W$ is Fredholm. It is injective by Lemma 2.2. Since the range of $W$ is closed, there is a number $c>0$ such that

$\|W f\|_{2} \geq c\|f\|_{2}$ for all $f \in L^{2}(\mu)$.

In particular choosing $f=\chi_{E}$ and $\delta=\frac{c^{2}}{n}$, where $E \in \Sigma$ and $\mu(E)<\infty$, we obtain

$$
\begin{gathered}
c^{2} \mu(E) \leq|| W \chi_{E} \|_{2}^{2} \\
\leq \int_{X}\left|\sum_{i=1}^{n} u_{i} \chi_{E} o \varphi_{i}\right| d \mu \\
\leq n^{p-1} \sum_{i=1}^{n}\left|u_{i}\right|^{2} \chi_{E} o \varphi_{i} d \mu \\
=n^{p-1} \int_{X} J \chi_{E} d \mu .
\end{gathered}
$$

(b) Assume that there is some $\delta>0$ such that $J \geq \delta \mu-$ a.e, on $\operatorname{Coz} J=\{x \in X ; J(x) \neq 0\}$. We know that $\operatorname{ker} W \subseteq L_{\mid X \backslash \text { Coz } J}^{2}(\mu) \quad, \quad$ where $L_{X \backslash \text { Coz J }}^{2}(\mu)=$ $\{f ; f(\operatorname{Coz} J)=0\}$. Since $W^{*} W f=J f$ for every $f \in L^{2}(\mu)$,

Obviously $W$ is injective and so is semi-Fredholm. 


\section{Conclusion}

In this paper, determine the completely continuus, M-weakly compactness and Fredholmness of finite sum of weight composition operators is discussed. Firstly, the necessary condition for completely contininuuse and M-weakly compactness and the necessary and sufficient conditions for compactness of finite sum of weight composition operators are obtained. Then, discuss Fredholmness of this operators on $L^{p}$-spaces and finite sum of weight Frobeniuse-Perron operators on $L^{1}$-spaces.

\section{References}

[1] A. Lambert, Leealising set for sigmaalgebra and related point transformaions, Proc. Roy. Soz. Endinburgh Seci. A, 118 (1991), $111-118$.

[2] A. Lambert, Operator algebras related to measure preserving transformations of finite order, Rocly Mountain J. Math., 14 (1984), 341-349.

[3] Lo, Ching On, Compact anf Fredholm weighted composition operators, A Thesis Submitted to Hong-Kong university (2010).

[4] P. Meyer - Nieberg, Uber Kllassen schwach kompakter Operatoren in Banachverbanden, Math. Z. Numer. Anal. (138), (1974), 145-159.

[5] R. K. Singh and J. S. Manhas, Composition operators on function spaces, North Holiand Math. Studies 179, Amsterdam (1993).
[6] H. Takagi, Compact weighted operators on $L^{p}$, Proc. Amer. Math. Soc., 116 (1992), 501-511.

[7] Y. Staremi, Unbounded weighted conditional expectation operators, Complex Anal. Oper. Theory, 10 (2016), 567-580.

[8] Y. Staremi and Jabbarzadeh, Weighted Lambert type operatots on $L^{p}$-spaces, Oper Matric., 7 (1) (2013), 101-116.

[9] M. R. Jabbarzadeh and Ystaremi, Essential norm of substitution operators on $L^{p}(\mu)$-spaces Indian J. Pure Appl. Math., 43 (3) (2012), 263-278.

[10] K. Narita and H. Takagi, Compact composition operators between $L^{p}(\mu)$-spaces, Harmonic analytic function spaces and linear operators, Kyoto University Research Hnstitute for Mathematic Sciences Kokyuroku, 1049 (1998), 129-136 (Japanese).

[11] E. A. Nordgen, Composition operators on Hilbert spaces, Lecture Note in Math., Springer Berlin, Vol 693 (1978) 37-63.

[12] S. K. Parrott, Weighted translation operators, Thesis, University of Machigan Ann Albur, 1965.

[13] M. M. Rao, Conditional measure and applications, Marcel Dekker, New York, 1993.

[14] J. C. Rho, J. K. Yoo (E)- super Decomposable operator, 1993.

[15] S. Shamsi Gamchi, A. Ebadain, A. Alishahi, Basic properties of finite sum of weighted composition operators 2017. 